\title{
Benefits of Combined Mental and Physical Training in Learning a Complex Motor Skill in Basketball
}

\author{
Andrea Gaggioli, Luca Morganti, Maurizio Mondoni, Alessandro Antonietti \\ Università Cattolica del Sacro Cuore, Milano, Italy \\ Email: lucafmorganti@gmail.com
}

Received June $28^{\text {th }}$, 2013; revised July $26^{\text {th }}$, 2013; accepted August $17^{\text {th }}, 2013$

\begin{abstract}
Copyright (c) 2013 Andrea Gaggioli et al. This is an open access article distributed under the Creative Commons Attribution License, which permits unrestricted use, distribution, and reproduction in any medium, provided the original work is properly cited.
\end{abstract}

\begin{abstract}
Previous research in sport psychology and rehabilitation suggests that training with motor imagery (mental practice) is a potentially effective strategy to improve motor performance. The goal of the present study was to investigate the benefits of combining mental and physical training in learning a complex motor skill in basketball (the lay-up shot). To this end, sixty female university students were randomly assigned to either mental practice with physical training, or physical training alone. Motor performance was assessed before and after a four-week training period. To assess motor learning performance, a video analysis was carried out by three independent raters on the motor task before and after the training in both conditions. Results showed that mental practice condition improved coordination and movement accuracy, suggesting the potential effectiveness of this approach in training complex motor skills; furthermore, findings indicate the feasibility and accuracy of using video-based analysis of movement in the assessment of motor performance improvements.
\end{abstract}

Keywords: Motor Learning; Motor Imagery; Mental Practice; Sport Performance; Complex Motor Skills

\section{Introduction}

Motor imagery refers to the mental simulation of a movement without its physical execution. This process has been widely used in combination with physical practice to improve performance (for reviews see, Driskell, 1994; Allami et al., 2007; Schuster et al., 2011). Athletes often deal with new skills to be learned and in several circumstances an incorrect execution of a skill can result in a fault given to the athlete; in team sports, an error by one player can affect the whole group (e.g., travelling in basketball is punished by stopping the game and giving the ball to the opponent team). It has been proposed that mental practice with motor imagery can enhance motor learning process by allowing the explicit representation of different stages of the action (Jeannerod \& Decety, 1995; Sherwood \& Lee, 2003). More specifically, the mental representation of the movement can be used during mental simulation to cue the learner on temporal and spatial elements of the skill. By rehearsing this representation, the learner can use this information to monitor and improve the physical performance of the skill (Murphy \& Jowdy, 1992). Starting from this background, the main objective of this study was to investigate the efficacy of mental practice to improve learning of a complex motor skill in basketball. The hypothesis formulated is that supplementing physical practice with mental practice improves specific components of a complex motor skill. Although evidence in favour of this hypothesis has been provided in several sports (Driskell et al., 1994; Cumming \& Williams, 2012), efficacy of mental practice with motor imagery on a closed motor task (in this case, lay-up shot) has been less investigated. Further, this study aimed at evaluating the feasibility and accuracy of a new methodology to assess motor learning performance in sports. This approach is based on multiple raters' evaluation of videorecorded movements along different dimensions.

\section{Mental Practice with Motor Imagery}

Richardson (1967) refers to mental practice as "the mental rehearsal of a task without any muscular movement” (in Olsson, 2008: p.133). Other recurring terms in the mental practice literature are "mental preparation" and "motor imagery". Mental preparation is a broad term describing all the mental techniques that an athlete might use during training sessions, whereas "motor imagery" is more specifically defined as the mental rehearsal of a movement in absence of a gross muscular activetion (Jeannerod, 1994). A further distinction is made between internal (or "kinaesthetic") and external (or "visual”) imagery (Lang, 1979). When taking an internal perspective, the subject imagines to execute the task from a first-person, body-centered frame of reference, by focusing on simulated perceptions and sensations. In the external perspective, the movement is imagined as if the subject were watching someone performing the task, like in a movie. According to Jeannerod (1994), however, only the internal perspective should be regarded as motor imagery, while the external perspective is more related to visual imagery. In particular, internal motor imagery features a kinaesthetic component that helps the subject to really "feel" the imagined movement, including not only the visual, but also the spatial and kinaesthetic characteristics of the movement. Although no real movement is performed during an imagery task, motor imagery and physical execution are characterized by common functional features. For example, the time taken to 
imagine the movement is correlated with the time needed to actually execute it (Decety et al., 1989): the link between motor imagery duration and actual motor action duration is also maintained following specific training aimed at improving the speed of the execution (Louis et al., 2008). Furthermore, the imagination of effortful action activates similar physiological changes to the actual execution of movements (e.g., increase in heart and respiratory rates; Decety et al., 1991). There is also evidence that physical constraints of the movement affect both real and imagined execution (Roure et al., 1998).

At the neurological level, compelling evidence exists that the imagination and the execution of the movement share common neuronal substrates (Karni, 1995; Jackson, 2003; Miller, 2010). Drawing on this compelling evidence of the functional and neurophysiological relationship between imagined and actual movements, several authors have proposed mental practice with motor imagery as an effective strategy to improve motor learning (Feltz \& Landers, 1983; Driskell et al., 1994; Guillot \& Collet, 2008; Schuster et al., 2011). The efficacy of this approach in improving performance of motor function has also been investigated in neurological rehabilitation in combination with physiotherapy, with promising results (Lafleur et al., 2002; Gaggioli et al., 2006; Sun et al., 2013).

\section{Applications of Mental Practice with Motor Imagery}

\section{In Sport}

Mental practice with motor imagery has been applied in different sport disciplines, showing benefits in speed, performance accuracy, muscle strength, movement dynamics, and motor skill performance (for a review, see Taktek, 2004). The specialized literature provides also specific indications about how motor imagery training can be optimized (Driskell et al., 1994). As concerns the relative effectiveness of external and internal (i.e. kinaesthetic) imagery in sport applications, Hardy and Callow (1999) investigated this issue in karate. They found that external imagery was more effective in task learning, whereas internal imagery was more effective in athletes with greater level of expertise, especially with tasks involving a complex series of movements and different body parts (White \& Hardy, 1995). Further, the efficacy of mental practice with motor imagery seems greater when it is applied to close as opposed to open sport skills (Coelho, 2007). In sports involving closed skills it is easier to figure out the real movement that will be performed. In open sports, conversely, athletes often have to deal with different factors that may prevent the correct execution of the task and affect the course of action. In general, most of individual sports involve closed skills, whereas most team sports involve open skills.

Starting from this background, the goal of the present study was to test the effectiveness of combined mental and physical practice against physical practice alone in learning a complex motor task in basketball (lay-up shot). We selected the lay-up shot because it has a well-coded structure and accuracy is very important for its optimal execution. A mistake during the feet tapping phase, for example, may cause a foul being called to the player: each athlete has to learn the exact sequence and execute the task by coordinating the movement of arms and knees, rhythmically preparing the ending jump to the basket. The lay-up shot is performed by leaping from below, laying the ball up near the basket, and using one hand to bounce it off the backboard and into the basket. Furthermore, the accuracy of the movement can be measured quantitatively by focusing on selected features of the motor task.

\section{Material and Methods}

\section{Participants}

Sixty female university students $(\mathrm{M}=21.25, \mathrm{SD}=2.73)$ volunteered to participate in the experiment: they all attended the first year of the course in Motor Sciences at Università Cattolica del Sacro Cuore in Milan, Italy. We decided to include only female participants to have a homogeneous sample in terms of gender. Participants were randomly assigned to either one of two conditions: mental practice combined with conventional physical training (MI) and physical training alone (C). Ten students were excluded from the analysis because they did not show up at the post-test $\left(\mathrm{t}_{2}\right)$. The group that performed the MI condition included 35 students $(\mathrm{M}=21.2$; $\mathrm{SD}=2.77)$; the control condition included 25 students $(M=21.3$; $S D=2.73)$. All participants were novice players and had not received previous training in lay-up shot technique. Participants received no payment or other forms of compensation for taking part into the research. Participants signed an informed consent module for this study. The training protocol and the research design have previously obtained the institutional approval of the Department of Psychology of Università Cattolica del Sacro Cuore in Milano.

\section{Measures}

Imagery ability. The "Vividness of Movement Imagery Questionnaire” (VMIQ: Isaac, Marks, \& Russell, 1986) in the Italian version adapted by Antonietti and Crespi (Antonietti \& Crespi, unpublished manuscript) was used to assess the participant's imagery ability. VMIQ is a 24-item questionnaire asking participants to imagine movements and rate their ability to imagine individual movements on a 5-point Likert scale ranging from 1 (very hard to see) to 5 (very easy to see). The VMIQ has been shown to have a good internal consistency for the visual subscale $(r=.95)$ and for the kinestetic subscale $(r=.97)$, with a test-retest coefficient over a 3-week period of $r=.76$ and of $r=.62$ over a 2-week period (Eton et al., 1998). The VMIQ has shown high ( $r=.81$; Isaac et al., 1986) to moderate ( $r=.60$; Eton et al., 1998) correlations with the Vividness of Visual Imagery Questionnaire (VVIQ; Marks, 1973) but low correlations with Movement Imagery Questionnaire $(r=.58$; Hall \& Martin, 1997). No participants were excluded on the basis of their VMIQ score (see Results section).

Lay-up shot. The lay-up shot performance was evaluated through 5-point Likert scales ( 1 = very poor, 5 = very good), which assessed five features of the movement: "Fluidity", "Rhythm”, “Coordination”, “Step Accuracy” and "Balance Landing”. Higher values in "Fluidity" indicate a smooth movement, without interruptions and variations in speed. "Rhythm" indicates the presence of a rhythmic component in the required steps of the motor sequence. "Coordination" indicates the player's ability to control and correctly move all the parts of the body required by the task: specifically for the lay-up shot, coordination between knees and arms is evaluated. "Step Accuracy" refers to the player's ability alternating steps during the execution of the movement, following the right timing and the right sequence of feet hitting the floor: a balanced posture is also required. Finally, "Balance Landing” focuses on the final 
phase of the movement, when the player has to keep the balance after the shot, so that the player can immediately take part to the next action.

\section{Materials}

The motor imagery instructions script was encoded as Mp3 audio file and then stored on a compact disc: the audio track lasted 74 seconds. The script traces the lay-up shot from an internal perspective: the athlete was instructed to recollect the real sensations of the movement. The script used for the experiment was as follows:

"Imagine that you are on the basketball field with the basket right in front of you. You receive the ball and hold it in your hands. By holding it tight, you begin your movement with a right foot step. Then you perform a quick step by moving the left and then the right foot. You feel the speed of your movement starting from feet and spreading all along your body. The ball is firmly settled on your body's side far from a possible defender's tackle. The horizontal strength that moves you is now loaded on the left foot that switches it vertical. You jump as high as you can feel your body fly. You flex your right knee and clearly feel the extended left leg lift off the floor just before the shot. You stare at the basket and complete the action by shooting the ball through a wrist circular movement, before landing with both feet on the court”.

Some key elements of the script helped participant's work: for example, each athlete was asked to imagine from an internal perspective through the use of the second-person singular. This was aimed at enhancing the athlete's involvement in the task and it can help him/her through a non-familiar kind of practice. Furthermore, words have been chosen to let the participant feel the movement like if he/she is performing it: the script is not just a bare description of the correct movement.

\section{Procedure}

The study consisted in three consecutive phases: pre-training, training and post-training. In the pre-training phase, a baseline measure was obtained by asking participants to execute five lay-up shots $\left(\mathrm{t}_{1}\right)$ while the experimenter video-recorded their performance. In the following, training phase, players in the MI group received a Mp3 file containing audio instructions which were designed to facilitate mental rehearsal of the lay-up shot. As suggested by previous research (Olsson, 2008), participants were instructed to mentally practice three times a week. Each mental practice session consisted of five repetitions and lasted 10 minutes for a total amount of 30 minutes per week. In order to monitor compliance, participants were asked to fill a report after each mental training session in which they described their feelings during mental rehearsal.

Participants assigned to the control group received half-anhour physical training, two times per week for four weeks, totalizing four hours of physical training (the same amount of physical training was received by participants assigned to the MI group). In the post-training phase $\left(\mathrm{t}_{2}\right)$, each participant performed a second series of five lay-up shots and performance was again videotaped by the experimenter. All participants were right-handed.

\section{Results}

\section{Video Analysis}

Participants performance was video-taped during the execution of the motor task before and after training. After, videos were collected and post-produced to obtain individual clips for each lay-out shot performed by participants. Next, these clips were recorded in random order on a DVD. The DVD was sent to three professional basketball trainers (all males, mean age $=$ 41.5 years) who were asked to rate each single lay-out shot included in the DVD (only once at time). The three judges had extensive experience in training and evaluating young basketball players and received no monetary or other form of compensation for their evaluation. The DVD instructions included no indication concerning the scope of the research or the type of training that was received by participants. Evaluators provided their ratings on an Excel form, which was sent back per email to the experimenter.

In order to test inter-rater agreement, Cronbach alpha values were calculated: high consistency (values from .83 to .87) emerged for an all the measures of the lay-up shot except for "Balance Landing", that met lower agreement $\left(\mathrm{t}_{1}=.65, \mathrm{t}_{2}\right.$ $=.55)$.

Table 1 reports the mean improvement $\left(t_{2}-t_{1}\right)$ for each measure. The mental practice group showed greater improvement in performance than the control group.

Five repeated-measures 2 (MI vs. C) X $2\left(t_{1}\right.$ vs. $t_{2}$ ) ANOVAs were computed to test the statistical significance of the differences observed in the two conditions. Results of the ANOVA showed a significant effect of training condition on "Coordination” $\left[\mathrm{F}(1,58)=5.603, p<.05, \eta^{2}=.088\right]$ and an almost significant effect of training on "Step Accuracy" $[F(1,58)=3.724$, $\left.p=.059, \eta^{2}=.060\right]$, whereas the other three features of the movement ("Fluidity", "Rhythm" and "Balance Landing") did not significantly differ between the two conditions. Descriptive statistics for "Coordination" and "Step Accuracy" are reported in Table 2. Figure 1 shows the interaction effect between Group and Time for "Coordination".

\section{Vividness of Movement Imagery Questionnaire}

Further analyses have been conducted to assess any correla-

Table 1.

Mean improvement $\left(\mathrm{t}_{2}-\mathrm{t}_{1}\right)$ on selected performance measures.

\begin{tabular}{cccccc}
\hline & Fluidity & Rhythm & $\begin{array}{c}\text { Step } \\
\text { Accuracy }\end{array}$ & Coordination & $\begin{array}{c}\text { Balance } \\
\text { Landing }\end{array}$ \\
\hline MI & 0.24 & 0.25 & 0.34 & 0.35 & 0.26 \\
C & 0.11 & 0.12 & 0.03 & 0.09 & 0.18 \\
\hline
\end{tabular}

Table 2.

Coordination and step accuracy (Mean and SD).

\begin{tabular}{ccccc}
\hline & \multicolumn{2}{c}{ Coordination } & \multicolumn{2}{c}{ Step Accuracy } \\
\cline { 2 - 5 } & $\mathrm{t}_{1}$ & $\mathrm{t}_{2}$ & $\mathrm{t}_{1}$ & $\mathrm{t}_{2}$ \\
\hline $\mathrm{MI}$ & $2.85(0.94)$ & $3.20(0.86)$ & $2.90(1.07)$ & $3.24(0.97)$ \\
$\mathrm{C}$ & $2.53(0.55)$ & $2.63(0.64)$ & $2.65(0.68)$ & $2.68(0.76)$ \\
\hline
\end{tabular}




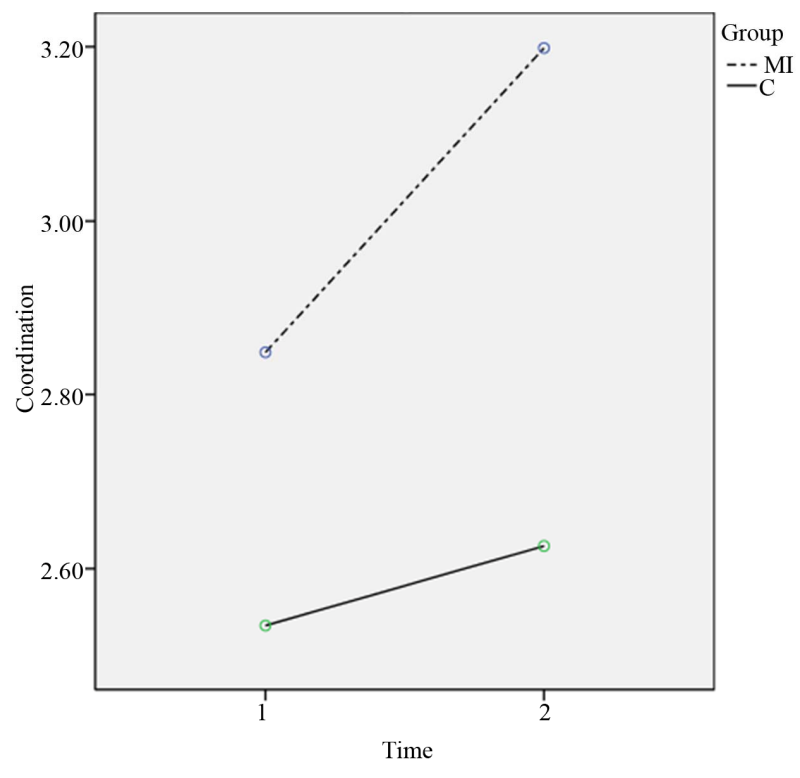

Figure 1.

Coordination $\mathrm{X}$ time interaction.

tions between VMIQ scores and performance improvement: the participants scored from 84 to 120 , with a mean value on the whole sample of 99.17. The sample was split into tertiles considering their imagery ability (VMIQ score), assessed before training. Pearson correlation coefficients with performance scores (the mean improvement between $t_{1}$ and $t_{2}$ for each features) were calculated. No significant correlations were found.

\section{Discussion}

Consistent with the main hypothesis of this study, findings indicate that combined mental and physical practice can improve performance of a closed sport task. These findings are partially in line with those obtained by related studies investigating motor imagery in basketball (Post et al., 2010; Lamirand \& Rainey, 1994; Savoy \& Beitel, 1996). Guillot et al. (2009) trained three attack movements with 10 national female basketball players: results showed that motor imagery improved motor performance, although mental practice was not found more effective than physical practice alone. Other research has indicated that imagery efficacy in basketball is enhanced when it is supported by visual cues (Hall \& Erffmeyer, 1983) or it is performed right before the match (Post et al., 2010). In the present study, the lay-up shot technique was examined with respect to several dimensions ("Fluidity”, "Rhythm”, "Step Accuracy”, "Coordination" and "Balance Landing"). Among these features, "Balance Landing” was the only feature showing relatively low agreement among judges. Since "Balance Landing” is a crucial indicator of the relationship between the individual movement and athlete's ability to start the next team action, this is a crucial feature to consider in future studies employing mental practice in basketball. The results showed a significant improvement in "Coordination", and in particular evaluators considered the coordination between arms and knees. This is a difficult challenge for novices, since not only they must learn different patterns of movement involving different body parts, but they also have to synchronize them. The imagery instructions script provided by our training protocol helped novices taking into account all the different parts of the task, and particularly how to associate them in order to perform an homogeneous movement. To our best knowledge, this is the first study showing a positive effect of motor imagery on coordination in learning a complex motor skill in sport: however, similar findings were obtained in the clinical context, in particular in the application of motor imagery in developmental coordination disorder (Wilson, 2002; Doussoulin \& Rehbein, 2011). The improvement observed in Step Accuracy, although close to significance, deserves also a comment. This feature is directly linked to the cognitive dimension of motor learning: the main challenge for an athlete learning the lay-up shot is represented by the acquisition of the right sequence of steps, whose difficulty is high because the final performance must be very quick. Once again, the imagery script guided the athlete to take into account that the three steps required are not only an abstract sequence nor simply a series of rules to follow. The perceptions recalled through the imagery script allowed the athlete to feel and consider the lay-up shot as the best and fastest way to reach the basket efficiently. The imagery script was rehearsed from an internal viewpoint, so that the participant was able to learn both the correct execution and the bodily sensations and feelings, i.e. the shift of body's weight while performing the task. Giving them a detailed script they could focus on, athletes better understood the sequence as a pattern that makes sense to the actual performance.

Secondly, we have introduced a novel methodology for evaluation of the motor skill performance, based on the analysis of the video-tapes of the target movement. Recent studies have attempted to assess motor imagery effects through self-reported measures, like the Standardized Basic and Combined Movements Scale (Dossoulin \& Rehbein, 2011) or the Sport Imagery Questionnaire (Hall et al., 1998). These studies aim at identifying the role of imagery training in improving psychological issues like self or collective efficacy (Shearer et al., 2009) or the imagery ability itself (Cumming et al., 2001). Other studies analyze the outcome from a mathematical perspective with a specific parametric analysis of distances and angles of the movements, e.g. in golf (Smith et al., 2008) and high jump (Olsson et al., 2008). A small number of researches in sport assess the final score of the movement or the number of successful attempts (Peynircioglu et al., 2000; Ploszay et al., 2006). However, we propose that video-analysis performed by independent raters represents a way of assessing motor performance which may reduce some of the bias usually associated with self-reports. This methodology allowed us to rate directly the performance in a simple way, besides the results highlight the specific features enhanced by the protocol instead of a general improvement.

However, it should be mentioned that this study had several limitations. First, the sample involved only female participants, limiting the generalizability of findings. Further, the sample included only students; therefore it is not clear to which extent these findings are also applicable to athletes.

A future goal is to investigate how to incorporate this newlydesigned imagery protocol in the training program of a team. Our training could be specifically addressed to the novices showing greater difficulties in performing a synchronized movement. For example, a coach could identify the specific strengths and weaknesses of each athlete, e.g., lack of coordination, to offer them specific motor imagery training, empowering the physical one practiced during team sessions with the help of a 
sport psychologist (or just scheduled by a specialist and then performed autonomously by the athlete). Future studies should also aim to investigate the real efficacy of mental training with younger athletes (Munroe-Chandler, 2007; Parker et al. 2009), taking into account also their ability of performing an imagery protocol based on internal perspective (Parker, 2011).

\section{Acknowledgements}

We wish to thank Michele Moscardin for registering the audio tracks.

\section{REFERENCES}

Allami, N., Paulignan, Y., Brovelli, A., \& Boussaud, D. (2007). Visuomotor learning with combination of different rates of motor imagery and physical practice. Experimental Brain Research, 184, 105-113. doi:10.1007/s00221-007-1086-X

Coelho, R. W., De Campos, W., Da Silva, S. G., Okazaki, F. H., \& Keller, B. (2007). Imagery intervention in open and closed tennis motor skill performance, Perceptual and Motor Skills, 105, 458-468.

Cumming, J. L., \& Ste-Marie, D. M. (2001). The cognitive and motivetional effects of imagery training: A matter of perspective. The Sport Psychologist, 15, 276-288.

Cumming, J., \& Williams, S. E. (2012). Imagery: The role of imagery in performance. In S. Murphy (Ed.), Handbook of sport and performance psychology (pp. 213-232). New York, NY: Oxford University Press. doi:10.1093/oxfordhb/9780199731763.013.0011

Decety, J., Jeannerod, M., Germain, M., \& Pastene, J. (1991). Vegetative response during imagined movement is proportional to mental effort. Behavioural Brain Research, 42, 1-5. doi:10.1016/S0166-4328(05)80033-6

Decety, J., Jeannerod, M., \& Prablanc, C. (1989).The timing of mentally represented actions. Behavioural Brain Research, 34, 35-42. doi:10.1016/S0166-4328(89)80088-9

Doussoulin, A., \& Rehbein, L. (2011). Motor imagery as a tool for skill training in children, Motricidade, 7, 37-43.

Driskell, J., Copper, C., \& Moran, A. (1994). Does mental practice enhance performance? Journal of Applied Psychology, 79, 481-492. doi:10.1037/0021-9010.79.4.481

Eton, D. T., Gilner, F. H., \& Munz, D. C. (1998). The measurement of imagery vividness: A test of the reliability and validity of the vividness of visual imagery questionnaire and the vividness of movement imagery questionnaire. Journal of Mental Imagery, 22, 125136.

Feltz, D. L., \& Landers, D. M. (1983). The effects of mental practice on motor skill learning and performance: A meta-analysis. Journal of Sport Psychology, 5, 25-57.

Gaggioli, A., Meneghini, A., Morganti, F., Alcaniz, M., \& Riva, G. (2006). A strategy for computer-assisted mental practice in stroke rehabilitation. Neurorehabilitation and Neural Repair, 20, 503-507. doi:10.1177/1545968306290224

Guillot, A., Nadrowska, E., \& Collet, C. (2009). Using motor imagery to learn tactical movements in basketball. Journal of Sport Behavior, 32, 189-206.

Guillot, A., \& Collet, C. (2008). Construction of the motor imagery integrative model in sport: A review and theoretical investigation of motor imagery use. International Review of Sport and Exercise Psychology, 1, 31-44. doi:10.1080/17509840701823139

Hall, C. R., \& Martin, K. A. (1997). Measuring movement imagery abilities: A revision of the movement imagery questionnaire. Journal of Mental Imagery, 21, 143-154.

Hall, C. R., Mack, D., Paivio, A., \& Hausenblas, H. (1998). Imagery use by athletes: Development of the sport imagery questionnaire. International Journal of Sport Psychology, 29, 73-89.

Hall, E., \& Erffmeyer, E. S. (1983). The effect of visuo-motor behavior rehearsal with videotaped modeling on free throw accuracy of intercollegiate female basketball players. Journal of Sport Psychology, 5,
343-346.

Hardy, L., \& Callow, N. (1999). Efficacy of external and internal imagery perspectives for the enhancement of performance in tasks in which form is important. Journal of Sport and Exercise Psychology, 21, 95-112.

Isaac, A., Marks, D., \& Russell, D. (1986). An instrument for assessing imagery of movement: The Vividness of Movement Imagery Questionnaire (VMIQ). Journal of Mental Imagery, 10, 23-30.

Jackson, P. L., Lafleur, M. F., Malouin, F., Richards, C. L., \& Doyon, J. (2003). Functional cerebral reorganization following motor sequence learning through mental practice with motor imagery. Neuroimage, 20, 1171-1180. doi:10.1016/S1053-8119(03)00369-0

Jeannerod, M. (1994). The representing brain: Neural correlates of motor intention and imagery. Behavioural and Brain Sciences, 17, 187-245. doi:10.1017/S0140525X00034026

Jeannerod, M., \& Decety, J. (1995). Mental motor imagery: A window into the representational stages of action. Current Opinion in Neurobiology, 5, 727-732.

Karni, A., Meyer, G., Jezzard, P., Adams, M. M., Turner, R., \& Ungerleider, L. G. (1995). Functional MRI evidence for adult motor cortex plasticity during motor skill learning, Nature, 377, 155-158. doi:10.1038/377155a0

Lafleur, M. F., Jackson, P. L., Malouin, F., Richards, C. L., Evans, A. C., \& Doyon, J. (2002). Motor learning produces parallel dynamic functional changes during the execution and imagination of sequential foot movements. NeuroImage, 16, 142-157. doi:10.1006/nimg.2001.1048

Lamirand, M., \& Rainey, D. (1994). Mental imagery, relaxation, and accuracy of basketball foul shooting, Perceptual and Motor Skills, 78, 1229-1230. doi:10.2466/pms.1994.78.3c.1229

Lang, P. J. (1979). A bio-informational theory of emotional imagery. Psychophysiology, 16, 495-512. doi:10.1111/j.1469-8986.1979.tb01511.x

Louis, M., Guillot, A., Maton, S., Doyon, C., \& Collet, C. (2008). Effect of imagined movement speed on subsequent motor performance. Journal of Motor Behavior, 40, 117-132. doi:10.3200/JMBR.40.2.117-132

Marks, D. F. (1973). Visual imagery differences in the recall of pictures. British Journal of Psychology, 64, 17-24. doi:10.1111/j.2044-8295.1973.tb01322.x

Miller, K. J., Schalk, G., Fetz, E., den Nijs, M., Ojemann, J., \& Rao, R. (2010). Cortical activity during motor execution, motor imagery, and imagery-based online feedback. Proceedings of the National Academy of Sciences, 107, 4430-4435.

Munroe-Chandler, K. J., Hall, C. R., Fishburne, G. J., \& Strachan, L. (2007). Where, when and why young athletes use imagery: An examination of developmental differences. Research Quarterly for Exercise and Sport, 78, 103-116. doi:10.1080/02701367.2007.10599408

Murphy, S. M., \& Jowdy, D. P. (1992). Imagery and mental practice. In T. S. Horn (Ed.), Advances in sport psychology (pp. 221-225). Champaign, IL: Human Kinetics.

Olsson, C. J., Jonsson, B., \& Nyberg, L. (2008). Internal imagery training in active high jumpers. Scandinavian Journal of Psychology, 49, 133-140. doi:10.1111/j.1467-9450.2008.00625.x

Parker, J. K., \& Lovell, G. (2009). Characteristics affecting the use of imagery: A youth sports academy study. Journal of Imagery Research in Sport and Physical Activity, 4, 1-15.

Parker, J. K., \& Lovell, G. (2011). The influence of experience upon imagery perspectives in adolescent sport performers. Journal of Imagery Research in Sport and Physical Activity, 6, Article 1.

Peynircioglu, Z. F., Thompson, J. L. W., \& Tanielan, T. B. (2000). Improvement strategies in free-throw shooting and grip-strength task. The Journal of General Psychology, 127, 145-156. doi:10.1080/00221300009598574

Ploszay, A. J., Gentner, N. B., Skinner, C. H., \& Wrisberg, C. A. (2006). The effects of multisensory imagery in conjunction with physical movement rehearsal on golf putting performance. Journal of Behavioral Education, 15, 247-255. doi:10.1007/s10864-006-9034-6

Post, P. G., Wrisberg, C. A., \& Mullins, S. (2010). A field test of the 


\section{A. GAGGIOLI ET AL.}

influence of pre-game imagery on basketball free throw shooting. Journal of Imagery Research in Sport and Physical Activity, 5, Article 2.

Richardson, A. (1967). Mental practice: A review and discussion (Part II). Research Quarterly, 38, 263-273.

Roure, R., Collet, C., Deschaumes-Molinaro, C., Dittmar, A., Rada, H., Delhomme, G., \& Vernet-Maury, E. (1998). Autonomic nervous system responses correlate with mental rehearsal in volleyball training. European Journal of Applied Physiology, 78, 99-108. doi:10.1007/s004210050393

Savoy, C., \& Beitel, P. A. (1996). Mental imagery for basketball. International Journal of Sport Psychology, 27, 454-462.

Schuster, C., Kilfiker, R., Amft, O., Scheidhauer, A., Andrews, B., Butler, J., Kischka, U., \& Ettlin, T. (2011). Best practice for motor imagery: A systematic literature review on motor imagery training elements in five different disciplines. BMC Medicine, 9, 75. doi:10.1186/1741-7015-9-75

Shea, C. H., Wright, D. L., Wulf, G., \& Whitacre, C. (2000). Physical and observational practice afford unique learning opportunities. Journal of Motor Behavior, 32, 27-36. doi:10.1080/00222890009601357

Shearer, D., Mellalieu, S., Shearer, C., \& Roderique-Davies, G. (2009). The effects of a video-aided imagery intervention upon collective efficacy in an international paralympic wheelchair basketball team. Journal of Imagery Research in Sport and Physical Activity, 4, 1-25. doi:10.2202/1932-0191.1039

Sherwood, D. E., \& Lee, T. D. (2003). Schema theory: Critical review and implications for the role of cognition in a new theory of motor learning. Research Quarterly for Exercise and Sport, 74, 376-382. doi:10.1080/02701367.2003.10609107

Smith, D., Wright, C. J., \& Cantwell, C. (2008). Beating the bunker: The effect of PETTLEP imagery on golf bunker shot performance. Research Quarterly for Exercise and Sport, 3, 386-391.

Sun, L., Yin, D., Zhu, Y., Fan, M., Zang, L., Wu, Y., Jia, J., Bai, Y., Zhu, B., \& Hu, Y. (2013). Cortical reorganization after motor imagery training in chronic stroke patients with severe motor impairment: A longitudinal fMRI study. Neuroradiology, 55, 913-925. doi:10.1007/s00234-013-1188-z

Taktek, K. (2004). The effects of mental imagery on the acquisition of motor skills and performance: A literature review with theoretical implications. Journal of Mental Imagery, 3, 79-114.

White, A., \& Hardy, L. (1995). Use of different imagery perspectives on the learning and performance of different motor skills. British Journal of Psychology, 86, 169-180. doi:10.1111/j.2044-8295.1995.tb02554.x

Wilson, P. H., Thomas, P. R., \& Maruff, P. (2002). Motor imagery training ameliorates motor clumsiness in children. Journal of Child Neurology, 17, 491-498. doi:10.1177/088307380201700704 